\title{
The optimization of medical X-ray images
}

\author{
Z. Garaguly*†, M. Kozlovszky*†, and L. Kovács*‡ \\ *John von Neumann Faculty of Informatics, Obuda University, Budapest, Hungary \\ $\dagger$ BioTech Research Center, University Innovation and Research Center, Obuda University, Hungary \\ $\ddagger$ Physiological Controls Group, University Innovation and Research Center, Obuda University, Hungary
}

\begin{abstract}
The current paper is concerned on one of the most important branch of medical picture modality, the X-ray. The spread of making digital X-ray images nowadays has great importance, because it has numerous advantages in contrast with the traditional image shooting process. However, some of its disadvantageous features means great restraining power, but these can be cured-even if not easily-in the world of informatics. Such disadvantage, for instance, is the picture definition, the contrast, bit-depth, and in case of digital technique, the human factor should be taken into consideration as well, because the human eye restricts the visualization in great extent. Regarding these problems, the article is about the introduction of such image optimization process, by which the usability of the $\mathrm{X}$-ray pictures can be corrected with improving the picture quality.
\end{abstract}

\section{INTRODUCTION}

With the optimization of the X-ray image, the digital X-ray images can be improved, therefore the represented tissue structures can be separated better. The mistakes, that emerge at the shooting, such as overdriving or inappropriate positioning, can be corrected posteriorly, so it is no necessary to take another shooting of the patient and burden his body. Furthermore, the diagnostician does not have to work with a low-quality picture, so a more precise diagnosis can be established using less time and energy.

This paper describes the analog and digital X-ray technology, and details how optimize the digital X-ray images. In the first part of the paper, a short overview of X-ray, and X-ray imaging is given. It is followed by an introduction to digital X-ray technology and a discussion about the key issues of image optimization. Then we give a general overview about X-ray imaging, image optimization, and describe briefly how they are implemented. We also describe image optimization case studies focusing on the optimization problem.

\section{MATERiAls AND Methods}

\section{A. X-ray technology}

1) X-ray tube: The X-radiation is gained by the transformation of the electrons energy, which process is taken place in the X-ray tube. The quantity and quality of the X-radiation can be affected by electric quantities $(k V, m A)$ and the time as well. Thus the X-ray tube is an energy converter, which can transform the electric energy into two forms of energy: $\mathrm{X}$-ray and heat. Heat in this situation can be considered as a redundant byproduct. The basic assignment of the X-ray tube - which is regarded at the planning and formation of the tube - is that the X-ray emission should be maximal and the heat transmission should be optimal. The X-ray tube is a relatively simple electric device, which consists of two parts, the anode and the cathode. If we connect the X-ray tube into a circuit, the current passes from the cathode towards the anode, the electrons transmit their energy due to X-ray (and heat) emerges [1], [2].

2) Cathode: The cathode is the negative side (pole) of the X-ray tube. On the cathode the electron cloud emerges under the heat, due to high voltage the electrons move towards the anode, and the cathode construction focuses this electron current towards the appropriate part of the anode. The basic parts of the cathode are: the cathode filament, the focusing cup [1], [3].

3) Cathode filament: The cathode filament is a thin wolfram filament which is $0,1-0,2 \mathrm{~mm}$ diameter and this wolfram filament is $1-2 \mathrm{~mm}$ wide and constructs a $10-15 \mathrm{~mm}$ long roll. The cathode filament in the cathode is situated in the so-called focusing cup [1], [3].

4) The focusing cup: The focusing cup means the indentation of the cathode construction where the cathode filament is situated. It is usually made of nickel and its assignment derives from its name, to focus the moving electrons due to high voltage on the appropriate point of the anode [2], [4].

5) Anode: The anode is the positive side of the X-ray tube which has three functions: 1 . The target of the high-speed electrons coming from the anode; the X-ray photons place of emergence 2 . The part of the high-voltage current circuit 3 . It does the diversion of heat. Within the anode, the focus area is the part where the high-speed electrons suddenly arrest and Xray photons are about to emerge. The anode can be presented in two kind of organization in the X-ray tube: as a standing or a turning anode. Nowadays standing anode is only applied in low-power X-ray devices, so most of the appliances contain turning anode. The turning anode turns during exposition, in consequence, the focus area significantly increases [1], [4].

\section{B. The $X$-ray image}

With X-ray radiation the method of picture creation would be that the X-ray goes through the body or a part of the body and we project a shadow image on the appropriate receptor (projection). The visualization of the radiographic picture has two ways: the analogue and the digital. The analogue mode means the X-ray film, which should be illuminated by an external light source - depending on the blackening of the film - where from some areas of the film different amount of light photon can get through. The blackening of the film is in connection with the intensity of X-ray photons that have 
reached the film. The digital visualization occurs by monitors, where the radiographic picture is visualized by the light photon emergence, which implicitly correlates with the X-ray photon intensity reached by the detector as well [5].

During the emergence of the X-ray picture we encounter different contrasts. The emergence of the visible picture means the inter-transformation of different kinds of contrasts, and its last phase is the contrast that emerged after fixing the picture.

1) Contrast: In order to get an object visible on the $\mathrm{X}$ ray picture, it should be contrasted in the physical sense in comparison with the surrounding material or tissue. The physical contrast could mean density difference or chemical compositional (serial number) difference. If an object physically differs from its environment, it absorbs more or less $\mathrm{X}$-ray radiation in comparison with the surrounding texture with the same thickness, which results shadow in the X-ray. If the object absorbs less radiation (e.g. gases), then it appears with the negative shadow, thus as a dark area on the X-ray film [6].

The third factor, which affects the object contrast, is the object's thickness in the direction of the X-ray's trespassing. The object contrast is proportional with the object's density and thickness and it's product. The contrast derives from the difference in chemical composition, and is extremely sensitive to the photon energy of the X-ray [7].

The X-ray contrast means the contrast emerged from the examined patient's body on the still invisible picture, which derives from the expositional difference between each points of the picture. In case of a special object an important contrast rate is denoted by the expositional difference between the represented area by the object and its environment. This expositional difference means the background and the percentage rate correlated to the environment. The contrast emerges when the exposition which represents the object's area will be more or less than the environment's/background's exposition [1], [7].

The picture contrast is the contrast that appears on the visible picture. On the X-ray film or on the monitor, it means the density difference between each points of the picture. The quantity of the visible contrast is determined by the features of the X-ray contrast and the film contrast. There is further possibility on the digital picture/monitor to set the contrast, by which the final contrast of the picture can be modified [7].

On the above we have always examined only one formula's/object's and its environment's relation where the increasing of the contrast usually also increased the visibility of the formula/object. In most of the clinical situations a sole picture "contains" numerous formulas and anatomic structures. Specifically it could be a problem when the different formulas are situated in different areas of the body with different thickness and density. The chest and the chest shot make the problem felt, because in a chest shot not only the lungs but the spine is also imaged [8].

\section{Digital X-ray technology}

We call basic-corrected raw picture the directly achieved picture from the different digital image sources which already contains the basic corrections (bad-pixel correction, offset correction, gain correction). This can be a recording picture or a radioscopic picture. In X-ray technique, the X-ray pictures are always monochrome, however, in each applications these are artificially after-colored $(C T, M R)$. The typical picture size is $500 \times 500$ pixels to $4500 \times 4500$, and typically $8-16$ bit dense per pixel [9], [10].

The Flat Panel Detector (FPD), instead of traditional Xray film is a large $\mathrm{X}$-ray sensor which directly takes a digital picture of the incoming X-ray. On the entering surface, a large, typically $43 \times 43 \mathrm{~cm}$ scintillation visor can be found which transforms the incoming X-rays into visible light. Under it the typically $3 \mathrm{kx} 3 \mathrm{kx} 16$ bit light sensor matrix layer is situated, which directly transforms the light into electric energy and the emerged picture is transferred by cable or Wi-Fi connection to the controller PC. We distinguish static and dynamic FPDs. The static ones can only be used in recording (RAD) mode, whereas the dynamic can be also used in recording and in radiographic mode as well. The system applying flat panel detectors is called DR (Direct Radiography) system [10], [11].

Phosphor plate, instead of the traditional X-ray film is a phosphor-glazed sheet, which is taken to a cassette used for traditional films. The incoming X-rays creates elemental energy-accumulation in each phosphor-granule, that is how they store the picture. Afterwards the cassette should be taken into a reading unit which reads out the stored picture with laser beam and digital line scanner and transfers through Ethernet or USB connection to the connected computer. The phosphorplate systems are also called Computed Radiography (CR) systems [12].

\section{IMAGE OPTIMIZATION}

The image optimization module's main function is the optimization of the basic-corrected raw picture (radiographic living picture or recording picture) which is read out from the image source before visualization on the screen. The quality of the X-ray picture emerging at exposition (number of valuable bits, real spatial definition, content of noise, contrast) depends on numerous factors:

- The set parameters of X-ray $(k V, m A, m A s$, etc. $)$

- The technical condition of X-ray device (generator, tube, collimator, bars etc.)

- The positioning of the patient (direction, angle, etc.)

- The special constitutional features of the patient (size, muscularity, overweight, etc.)

- The anatomic specificity of the examined area (density, texture, occlusion of beams, reflection, homogeneity, etc)

The reasons for the necessity of image optimization:

- First of all, image optimization is needed because the human eye's definition of grey scale is 6-7 bit, therefore an average person can distinguish 100 kinds of shades of grey. Nowadays the digital X-ray images bit density is 
16, which is more than 65000 shades of grey. Actually the valuable average image content is smaller than that, but it is still more than the definition of the human eye. That is why the valuable image content in scanned digital raw image should be transformed into an 8 bit grey scale before visualization to be seen optimally on the monitor (Windowing) [13].

- Secondly, image optimization is necessary, because on the $\mathrm{X}$-ray picture the imaged anatomic area is nearly never homogeneous, where the different X-ray occlusive tissues (bones, muscles/sinews, soft parts, areas filled with liquid or gas) appears next to each other. As a result, if one tries to optimize the way that simultaneously every textural part can be seen on the picture the delicate details will be lost, or if one tries to optimize in the way that that the structure of the bones is visible, the soft part will be fallen out of the visible territory (This area will be too dark) [14].

- In addition, the recording of identical body parts (chest) should be visualized differently if the lungs would like to be examined on it, or the spine or the heart. It is important that what sort of examining code (aim) was the recording taken [15].

These problems are helped by method of "tissue harmonization" which artificially transforms the digital picture that the bigger X-ray occlusive (bone) and the smaller X-ray occlusive (soft tissues) areas details can be seen well simultaneously, for that we can see the Fig. 1. [8]
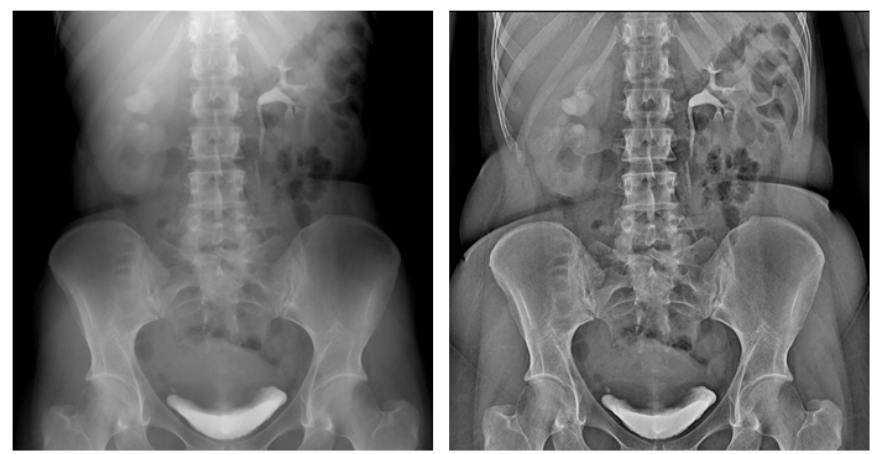

Fig. 1. Reference images.

The modules aim is to produce such optimized picture of any body part (APR) and relatively in any expositional circumstances (low-exposed or overexposed image) that for the medical expert each different X-ray occlusive textural structures can be appeared simultaneously with maximum in detail on the picture and without manual windowing, resulting in diagnosticable picture.

The steps of automatic image optimization presented in Fig. 2 are as follows.

- Filtering the bar noise on the raw picture.

Dissolving bar noise with automatic frequency recognition, the more modern flat panels do it on hardware.

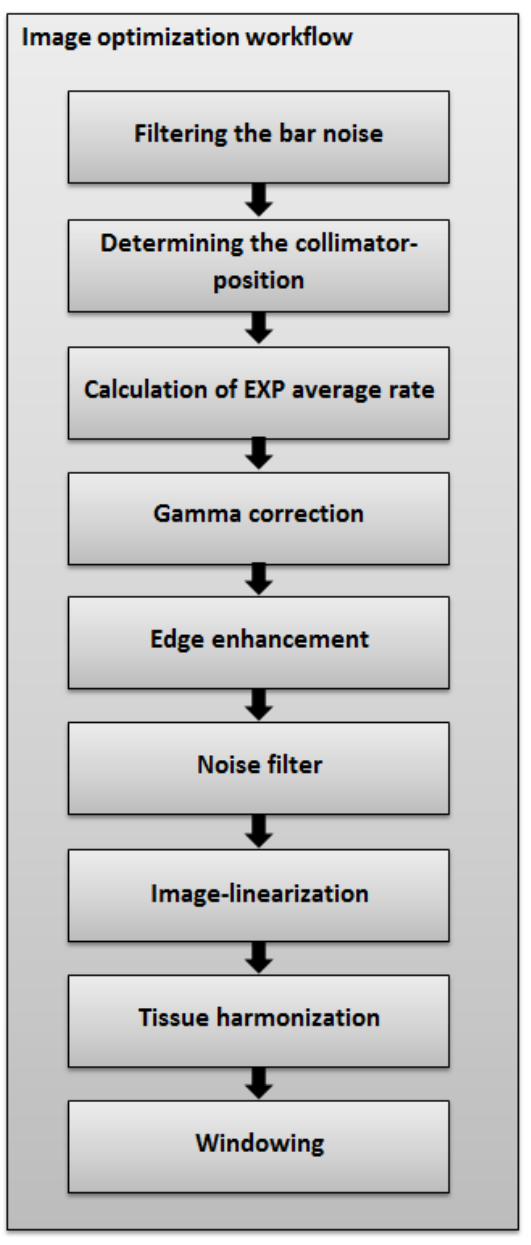

Fig. 2. Image optimization steps.

- Determining the collimator-position on the raw picture (software collimator).

Determining collimator position according to the examined body part.

- Calculation of EXP average rate.

Pictures with bad setting can be filtered with the calculation of this rate.

- Gamma correction on the raw picture.

Process of optimizing contrast rates. Formula: $V_{\text {out }}=$ $A V_{i n}^{\gamma}$, where $A$ is a constant and the $V_{i n}$ is the input and $V_{\text {out }}$ is the output values which are non-negative real values. Usually $A=1$, inputs and outputs typically range $0-1 . \gamma$ is the gamma value. If $\gamma<1$ is sometimes called an encoding gamma compression. If $\gamma>1$ is called a decoding gamma expansion.

- Edge enhancement with the given parameters.

To enhance the borders of tissue stuctures edges that these can be separated better from each other. During edge enhancement, the Canny edge detection has been applied [16].

- Noise filter with the given parameters.

Filtering noise induced by the flat panel detector. 
- APR body part dependent image-linearization. On the basis of the examined body part's data, histogram linearization is conducted.

- Tissue harmonization (ATH - Anatomical Tissue Harmonization, FTH - Fluoro Tissue Harmonization) according to the analysis of the picture, appliance of picture optimization in different parameters (noise-filter, edge enhancement, windowing) for the different areas of the picture

- Windowing for the optimal image visualization

\section{SOFTWARE STRUCTURE}

This chapter contains the realized steps and processes of the picture optimization process introduced in the former chapter. The revision of theoretical steps was needed, because there were no raw pictures available, which also means that there was no use of conducting operations with raw picture. The parameterization of each image processing steps was given manually, because the developed software was not a body partdependent image optimization software, but a general one. In its permanent phase, it can be used as a pre-optimization, but afterwards it can be expanded with a profiling unit, which contains the parameters belonging to the appropriate body parts. This way, there would be a profile assigned to every body part, and at the time of processing, only the profile we need should be chosen.

The modification of the mentioned steps order and construction was necessary for the realisation. All on the available Xray pictures bar noise was cleared out which was carried out by the making device itself. Determining the collimator position was not needed because in the permanent developmental phase we are not doing body part (APR) dependent image optimization but an ordinary one. After the gamma correction, noise filtering will be applied, before the edge enhancement. Furthermore, the tissue harmonization happened before edge enhancement, because it is practical to take it only when the appropriate tissue structure can be seen. On the basis of the above, a system design will be shown on Fig. 3 .

The operational process of the software starts with the income of the image. Then the image will be loaded, and afterwards the EXP average rate will be calculated. The next step is to conduct the gamma correction and the noise filtering. It is followed by the tissue harmonization and finally, the edge enhancement, and the windowing. After these operations are done, only the visualization and the saving is left behind. During an image optimization operation, these mentioned processes are taken place. On Fig. 4, a raw version of a chest shot can be seen on the left side, and its optimized version on the right, which was made by the own software.

\section{ACHIEVEMENTS}

The development of the application was launched as a test model, so that the development is on the level of a prototype. The result can be developed further, introducing and developing new processes. In the permanent state, it gives an ordinary harmonised picture as a result, on which every body part can

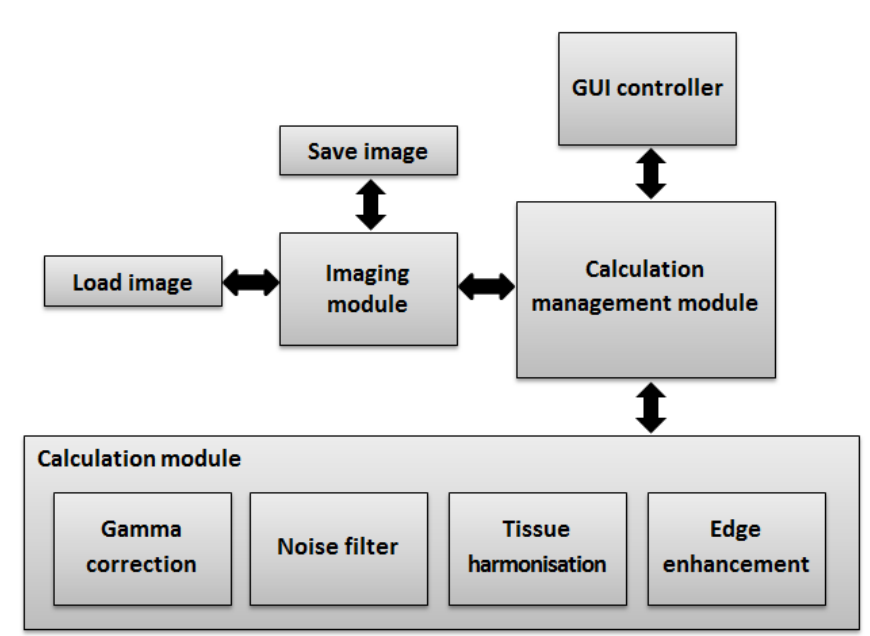

Fig. 3. Software system design.
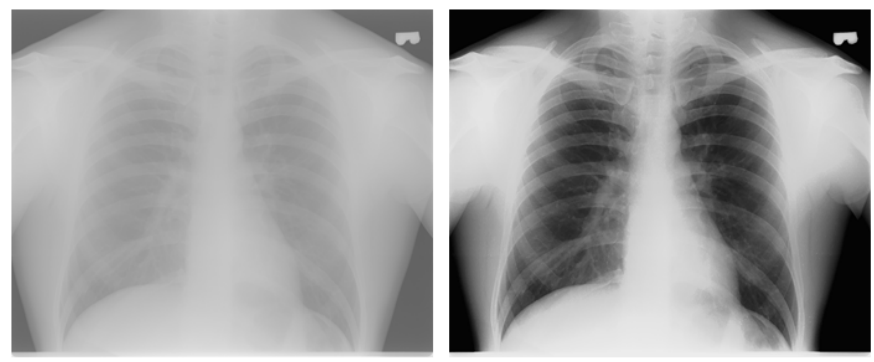

Fig. 4. Original chest X-ray image on the left and optimalized image on the right.

be seen, but none of the parts are filtered, or strengthened. With these images an ordinary routine assignment can be simplified, facilitated or removed, which is applied manually nowadays by doctors or X-ray assistants. It is a very difficult task to make such an optimization, which is appropriate for everyone and can be used better than the original raw picture, because a well-accustomed and well-diagnosable X-ray image is interpreted differently for everyone. We have tried to create an image which is readable and usable for everybody, in order to this, we were collaborating with an X-ray specialist. None of the optimization process was enhanced excessively, therefore the optimization software can be set for personal needs. Because of this feature, it is difficult to claim its goodness, particularly without measurable parameters. The time needed for the optimization process depends on the size of the image, but an approximately average images optimization lasts for 510 seconds. On Fig. 5, the original raw pictures can be seen on the left side , and on the right side, the harmonized pictures after the picture optimization.

\section{CONCLUSION}

Today, in the digital world, there is an increasing emphasis on digitalising and transforming the traditional analogue techniques, process in order to simplify, distinct and make processes faster. There is possibility to take digital X-ray 

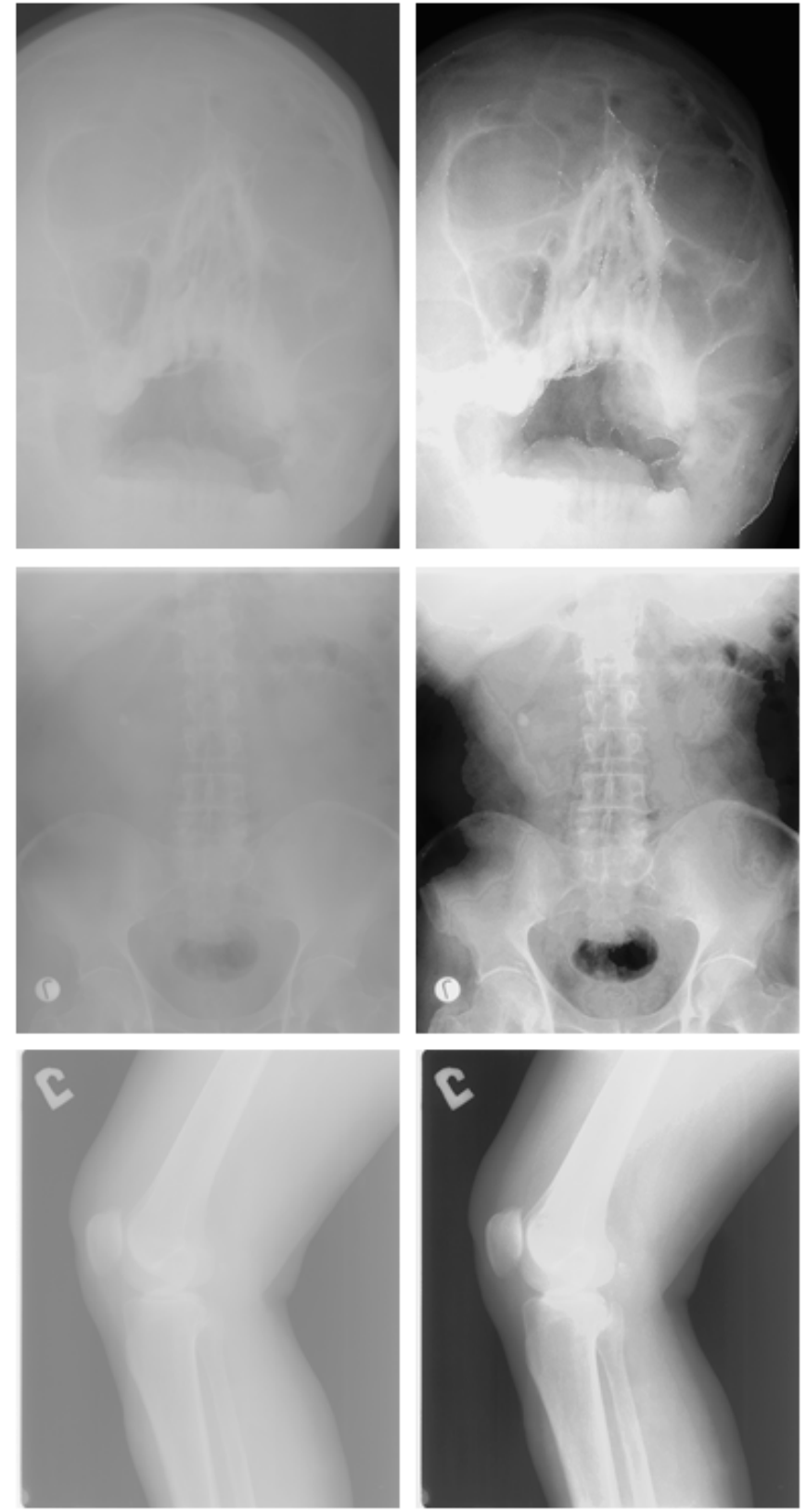

Fig. 5. Original X-ray images on the left and optimalized images on the right.

pictures instead of the traditional X-ray images. Its advantage is, that far away from the examination even on the other side of the world another doctor or specialist could also diagnose or give advice without sending the analogue picture to him physically. Its further advantage is that within a digital picture, there can be find a lot more information than on the analogue shot. By this way the digital pictures can be optimised posteriorly into different tissue types. It means that the shooting device should not be set at the time of the shooting to take a picture of the examined area with the appropriate contrast and definition but it can be done posteriorly on the digital X-ray image. As a result, the taken raw digital picture does not seem so high-qualified than the traditional picture, so it should be optimised on the basis of some kind of parameters. The image optimization means that those parts of the digital picture which cannot be seen by the human eye it becomes visible, and those which can be seen but it is only a disturbing noise or unwanted detail, it can be removed. For the appropriate optimization it should be known what kind of tissue structure is needed to be analysed. In the present realization, the aim was not to take such an optimized picture for examining a predetermined, body-part dependent tissue structure but to create a generally optimized view of the picture. The aim actually was to take such a picture which is competent for general diagnosis. This means that any kind of tissue structure is nor enhanced, but the image is optimized onto a general visible scale, in order to every object can be seen, but none of the parts will be over-enhanced, nor burn out (white) or disappear (black). Thus the pictures will be more usable and visible that without optimization. However if further examination from the specialist is needed, the hanging optimization of the body part and tissue structures will be necessary which is planned to be developed in the future.

\section{FURTHER WORK}

Further work is related to different anatomical areas (APR) according to its know features, usage of different optimizational process. Furthermore, the consideration of the quality of the emerged X-ray image can improve more in reaching the optimal picture. Its condition is that besides the image, the body part (APR) should be known from which the shooting was taken as well as the initial examinational code. The initial examinational code shows for what kind of tissue structure the examination is taken within a body part. Different settings should be applied, for instance, in case of a costal recording depending on the lungs or the spine need to be examined.

\section{ACKNOWLEDGMENTS}

This work was supported by the HP-SEE (HighPerformance Computing Infrastructure for South East Europe's Research Communities, under contract no. RI-261499) project and by the 3DHistech08 project (the Hungarian National Technology Programme, A1, Life sciences). Authors would like to thank PascalTeam Ltd. to provide us annotated samples for image processing and classification. The financial support of this work was from the University Innovation and Research Center, Obuda University, Hungary (EKIK). Ethical approval:"All procedures performed in studies involving human participants were in accordance with the ethical standards of the institutional and/or national research committee and with the 1964 Helsinki declaration and its later amendments or comparable ethical standards." 


\section{REFERENCES}

[1] P. Bogner, "The medical imaging physics" 2014. (in Hungarian)

[2] E. Spiller, "Soft X-Ray optics" 1994.

[3] B. H. Hasegawa, "The physics of medical x-ray imaging" Medical Physics Pub. 1990.

[4] C. J. Vyborny, "Image quality and the clinical radiographic examination" RadioGraphics Volume 17, Issue 2, 1997.

[5] Paul C. Johns, Martin J. Yaffe, "Theoretical optimization of dual-energy x-ray imaging with application to mammography" Med. Phys. 1985.

[6] Richard A. Ketcham, William D. Carlson, "Acquisition, optimization and interpretation of X-ray computed tomographic imagery: applications to the geosciences" Department of Geological Sciences, University of Texas at Austin USA 2001.

[7] Yasushi Nakano, Tomonori Gido, Satoshi Honda, Akihiro Maezawa Hideaki Wakamatsu, Takafumi Yanagita, "Improved computed radiography image quality from a BaFI:Eu photostimulable phosphor plate" Med. Phys. 2002.

[8] Elizabeth A. Krupinski, Mark B. Williams, Katherine Andriole, Keith J. Strauss, Kimberly Applegate, Margaret Wyattf, Sandra Bjork, J. Anthony Seibert, "Digital Radiography Image Quality: Image Processing and Display" American College of Radiology 2007.

[9] Spahn M, Strotzer M, Vlk M, "Digital radiography with a large-area, amorphous-silicon, flat-panel x-ray detector system" Invest Radiol 2000

[10] B. Abidi, M. Mitckes, M. A. Abidi, J. Liang, "Grayscale Enhancement Techniques for X-ray Images of Carry-on Luggage" Proc. of SPIE 6th International Conference on Quality Control by Artificial Vision 2003.

[11] Jeffrey H. Siewerdsen, David A. Jaffray, "Optimization of x-ray imaging geometry (with specific application to flat-panel cone-beam computed tomography)" Med. Phys. 2000

[12] R. S. Ledley, L. S. Rotolo, C. M. Park, T. J. Golab, "X-ray image enhancement" Georgetown University Medical Center, Washington 1980

[13] M. Ganten, B. Radeleff, A. Kampschulte, MD. Daniels, GW. Kauffmann, J. Hansmann, "Comparing image quality of flat-panel chest radiography with storage phosphor radiography and film-screen radiography" AJR Am J Roentgenol 2003.

[14] M. Uffmann, C. Schaefer-Prokop "Digital radiography: The balance between image quality and required radiation dose" European Journal of Radiology Volume 72, Issue 2, 2009.

[15] R. M. Koenker, "Digital radiography quality assurance: A technique for standardizing image appearance across CR and DR platforms" Appl Radiol., 2007.

[16] P. Bao, D. Zhang, Wu Xiaolin, "Canny edge detection enhancement by scale multiplication" Pattern Analysis and Machine Intelligence 2005. 